\title{
Open Creative Labs - Treffpunkte für Kreative?
}

\author{
Suntje Schmidt ${ }^{1}$ \\ Online publiziert: 6 . April 2020 \\ (c) Der/die Autor(en) 2020
}

\section{Zusammenfassung}

Vor dem Hintergrund einer zunehmenden räumlichen und sozialen Fragmentierung von Erwerbsarbeit hinterfragt der vorliegende Beitrag, inwiefern Open Creative Labs Treffpunkte für Kreativschaffende anbieten und wodurch diese Treffpunkte charakterisiert sind. Hierfür werden zunächst „Dritte Orte“ als Treffpunkte vorgestellt, strukturelle Wandlungsprozesse in Arbeitsmärkten skizziert und Open Creative Labs als neuartige physisch-soziale Arbeitsumgebungen vorgestellt. Dabei soll verdeutlicht werden, dass Open Creative Labs weit mehr als Treffpunkte in der Kreativwirtschaft darstellen.

\begin{abstract}
Against the background of the increasing spatial and social fragmentation of employment, this paper seeks to question in what way Open Creative Labs offer meeting venues for creatives, and how these venues are characterized. For this reason, the concept of "third places" as meeting venues is initially introduced, structural transformations in labor markets are outlined, and Open Creative Labs are envisaged as novel socio-material working environments. It should therefore be made clear that Open Creative Labs are much more than meeting venues for participants of the creative economy.
\end{abstract}

\section{Wo treffen für uns heute?}

Seit Mitte der 1990er-Jahre steigt die Zahl der Soloselbstständigen, also jener selbstständig tätigen Personen, die keine weiteren Beschäftigten in ihre Organisation einbinden, kontinuierlich an (Maier und Ivanov 2018). Eine ähnliche Entwicklung zeichnet sich ab in Bezug auf Selbstständige in freien Berufen, wie zum Beispiel in freien Kulturberufen und bei den selbstständigen Unternehmensberatern (IFB 2019). Dies bedeutet, dass der Anteil der kleinen unternehmerischen Einheiten ohne oder mit nur minimal ausgestatteten physisch-materiellen Umgebungen - wie beispielsweise voll ausgestatteten Büros einschließlich Sekretariatsfunktion - ebenfalls deutlich angestiegen ist. Zugleich beobachten wir vor allem in städtischen Agglomerationen seit den frühen 2000er-Jahren die zunehmende Einrichtung von Räumen und Orten, die von Selbstständigen, Start-up-Teams, Gründungswilligen, aber auch Bastlern, Experimentierfreudigen oder Hackern auf der Suche nach einer sinnstiftenden Tätigkeit genutzt werden. Hierzu zählen typischerweise Coworking Spaces, Fablabs, Makerspaces, Kreativlabs oder

Prof. Dr. Suntje Schmid

suntje.schmidt@geo.rwth-aachen.de

1 Lehrstuhl für Wirtschaftsgeographie, RWTH Aachen, Aachen, Deutschland offene Werkstätten - für die in diesem Beitrag der Oberbegriff Open Creative Labs als Dachbegriff verwendet wird. Vor allem die arbeitsbezogenen Labs werden überwiegend von Kreativschaffenden genutzt. Hierbei stellt sich die Frage, welchen Wert die Labs für diese Erwerbstätigen darstellen. Eine der Hauptthesen in der Literatur erklärt die Nachfrage nach den geteilten Arbeitsräumen mit dem Bedürfnis der Erwerbstätigen, die soziale Isolation im „Homeoffice“ zu umgehen.

Der vorliegende Beitrag beleuchtet vor diesem Hintergrund, inwiefern Open Creative Labs Treffpunkte für Kreativschaffende anbieten und wodurch diese Treffpunkte charakterisiert sind. Hierfür ist der Beitrag wie folgt aufgebaut: Zunächst soll kurz darauf eingegangen werden, durch welche Eigenschaften „Treffpunkte“ zu charakterisieren sind. Dabei orientiert sich der Beitrag insbesondere an dem Konzept der „Dritten Orte“ (Oldenburg 1997; Oldenburg und Brissett 1982). Anschließend soll kurz auf die Besonderheiten kreativer Tätigkeiten aus einer räumlichen Perspektive eingegangen werden, um danach die Funktionen von Open Creative Labs und deren Mehrwert für Kreativschaffende zu erläutern. Dabei soll verdeutlicht werden, dass Open Creative Labs weit mehr als Treffpunkte in der Kreativwirtschaft darstellen. 


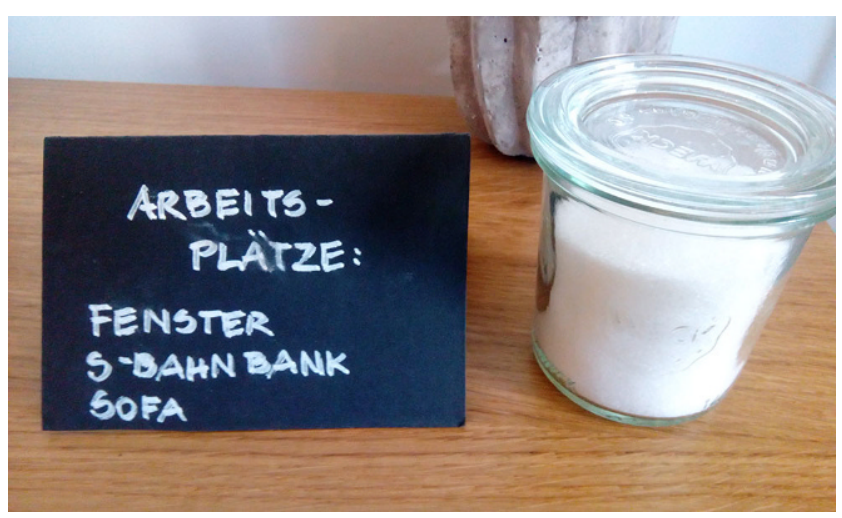

Abb. 1 Eingrenzung von Arbeitsplätzen in einem Berliner Café. (C) Suntje Schmidt)

\section{Treffpunkte als „Dritte Orte“}

Ray Oldenburg stellt „Dritte Orte“ als solche Lokalitäten vor, die keiner eindeutigen Funktion wie Wohnen („Erste Orte“) oder Arbeiten (,Zweite Orte“) zugeordnet werden können. Sie sind wichtige Knoten für den Aufbau und die Pflege sozialer Beziehungen, für gemeinschaftliches Miteinander, für das Aufeinandertreffen unterschiedlichster sozialer Gruppierungen und für ein Miteinander auch außerhalb etablierter Rollenmuster. „Dritte Orte“, wie zum Beispiel Cafés, Bibliotheken oder Parks, ermöglichen einen zeitlich befristeten Rückzug aus alltäglichen Rollenmustern und Aufgaben. Sie sind folglich zu verstehen als räumliche und soziale Kontexte außerhalb von Hierarchien, Abhängigkeiten oder konkreten Aufgaben, indem sie eine ungezwungene Atmosphäre bieten für einen weniger regelbehafteten, gar spielerischen Austausch (Oldenburg 1997). Dies verdeutlicht im Zusammenhang mit neuen Arbeitswelten auch Castells (2001) in der Beobachtung, dass in einer Bar im Silikon Valley mehr Wissen an einem Abend ausgetauscht wird als in akademischen Veranstaltungen in Stanford. Damit nehmen „Dritte Orte“ nicht nur eine soziale Funktion ein, sondern auch eine ökonomische, über die Wissen über räumliche und organisationale Grenzen hinweg diffundieren kann. Allerdings wäre eine derartige Diffusion nicht planbar, sondern eher zufällig, weil die soziale Mischung sich an diesen Orten zu unterschiedlichen Zeitpunkten immer andersartig und schwer vorhersehbar gestaltet. Während die Bar in Silicon Valley einen physischen Ort darstellt, der Menschen und Ideen miteinander verbindet, sind Bibliotheken „Dritte Orte“, in denen soziale Interaktionen zwischen Menschen sowie Menschen und verschiedenartigen Medien (z.B. Bücher, Onlineinformationen, zunehmend auch 3-D-Druck-Technologien) ermöglicht werden (Houghton et al. 2013). Die wohl wichtigste Funktion, die „Dritten Orten“ zugeschrieben wird, ist deren Rolle im Aufbau und Erhalt von Gemeinschaften und Gemeinschaftssinn (Houghton et al. 2013; Oldenburg 1997). Demzufolge kre- ieren „Dritte Orte“ einen gesellschaftlichen Mehrwert, weil sie dazu beitragen, Beziehungen innerhalb von Gemeinschaften zu stärken, Netzwerke auf- und auszubauen, lokale Gemeinschaften und Nachbarschaften einzubeziehen und Mehrwerte auch für unregelmäßige Nutzerinnen und Nutzer zu stiften.

Zusammenfassend lässt sich zunächst festhalten, dass „Dritte Orte“ Treffpunkte für verschiedenste soziale Gruppen darstellen, indem sie für diese Gruppen Interaktionsräume anbieten, einen gesellschaftlichen Mehrwert in Form von Gemeinschaftssinn stiften und Funktionen und Rollenmuster außerhalb stabiler Funktionen wie Wohnen oder Arbeiten ermöglichen.

Allerdings scheinen sich „Dritte Orte“ - besonders auffällig Cafés - immer stärker in Richtung einer Ökonomisierung und damit einer arbeitsbezogenen Funktion zu entwickeln. Hierfür reicht ein aufmerksamer Blick in innerstädtische Cafés von westlichen Großstädten, in denen zunehmend Menschen mit Kopfhörern vor ihren Laptops anzutreffen sind. Dies geschieht teilweise in einem Maße, dass CafébetreiberInnen beginnen, sich dagegen zu wehren, indem sie nur noch in ausgewiesenen Bereichen das Arbeiten gestatten (vgl. Abb. 1). Tatsächlich wird das Konzept der „Dritten Orte“ von einigen Autoren auf gemeinschaftliche Arbeitsorte übertragen, indem sie herausstellen, dass z.B. Coworking Spaces Kristallisationskerne für multiple soziale Praktiken einrichten und hierbei Experimentieren, spielerisches Miteinander, Lernen und Arbeiten gleichermaßen beinhalten (Waters-Lynch und Potts 2017). Demzufolge ermöglichen es Coworking Spaces, informelle soziale mit formellen, strukturierten und produktiven Funktionen zu verknüpfen. Dadurch würden vorteilhafte Interaktionen (wie zum Beispiel Sozialisieren, gemeinschaftliche Unterstützung, Mentoring, professionelles Netzwerken, Wissensaustausch und Zusammenarbeit) ermöglicht werden (Brown 2017). Schließlich tragen diese Orte ebenfalls dazu bei, ein Gefühl der Zugehörigkeit unter den Nutzerinnen und Nutzern aufzubauen und leisten damit einen wichtigen Beitrag zum Aufbau einer lokalen Gemeinschaft (Purnell und Cunningham 2017).

\section{Steigende Nachfrage nach Orten für Kreativarbeit}

Strukturelle Wandlungsprozesse in Arbeitsmärkten drücken sich einerseits aus in dem steigenden Anteil wissensintensiver und kreativer Tätigkeiten und andererseits in grundlegenden Veränderungen der institutionellen und organisatorischen Verfasstheit von Arbeit (Brown 2017; Florida 2011, 2017).

Trotz berechtigter kritischer Perspektiven zu den Thesen Richard Floridas in Bezug auf die kreative Klasse liegt 
einer seiner wesentlichen Beiträge darin, zu verdeutlichen, dass sich das Feld der Erwerbstätigen und ihrer wirtschaftlichen Aktivitäten deutlich verändert. Dies zeigt sich vor allem in der Zunahme von erwerbsbezogenen Tätigkeiten, die darauf ausgerichtet sind, neue Ideen, Technologien und kreative Inhalte $\mathrm{zu}$ generieren sowie komplexe Probleme zu lösen und dabei fachlich fundiertes Wissen und Erfahrungen gezielt einzusetzen (Florida 2002). Voraussetzung hierfür sind hochqualifizierte Ausbildungswege, die Fähigkeit, vorhandenes Wissen auf neuartige Weise zu verbinden und systematisch neues Wissen zu generieren. Derartige Tätigkeiten können nur begrenzt standardisiert werden, generieren individualisierte Produkte und Produktanpassungen oder wissensintensive Dienstleistungen und sind daher eng verbunden mit den individuellen Fähigkeiten und Kompetenzen der Erwerbstätigen. Dies zeigt sich einerseits an dem Anstieg von Berufen, die ein niedriges Substituierbarkeitspotenzial aufweisen und ein hohes Maß an Expertenwissen voraussetzen (Bundesagentur für Arbeit 2019). Andererseits zeigt sich dieser strukturelle Wandel der Arbeitsmärkte im Rückgang der sozialversicherungspflichtig Beschäftigten bei einem gleichzeitigen Anstieg von selbstständigen Tätigkeiten (Bundesagentur für Arbeit 2019). Hierzu zählen insbesondere die freien Berufe (wie freie Kulturberufe oder selbstständige Unternehmensberater; vgl. IFB 2019), Soloselbstständigkeiten (Maier und Ivanov 2018) bspw. in kreativ-schöpferischen Tätigkeiten (z. B. in den Teilmärkten der Kultur- und Kreativwirtschaft Architektur, Designwirtschaft, Werbung, Software- und Gamesindustrien; vgl. Söndermann und Strittmatter 2007) wie auch junge Unternehmensgründungen bspw. in Form von Start-ups.

Kreativ-schöpferische Tätigkeiten lösen sich deutlich von traditionellen organisatorischen und institutionellen Formen von Arbeit. Sie sind weniger von typischen Beschäftigungsverhältnissen (ausgedrückt in entfristeter sozialversicherungspflichtiger Vollzeitbeschäftigung) geprägt, sondern zeichnen sich durch zunehmend entgrenzte Formen des Arbeitens aus. Dies bedeutet einerseits, dass Erwerbstätige im Zusammenhang mit ihrer Beschäftigung hochgradig mobil sind, um ihre Leistungen zu erbringen. Andererseits drückt diese Charakterisierung der Arbeit aus, dass die Grenzen zwischen Freizeit und Arbeit zunehmend verschwimmen (Manske 2015) und damit auch die konkreten Orte, von denen aus gearbeitet wird. Zudem wird Arbeit insbesondere in wissensintensiven, kreativen Tätigkeiten vermehrt in temporären Strukturen wie Projekten, Netzwerken und Events organisiert (Grabher und Ibert 2011). So werden Leistungen in Form von Projekten - also Arbeitsstrukturen mit einem institutionalisierten Anfang und Ende - erbracht, für die entsprechende spezifische Verbünde eingerichtet werden, die die jeweils notwendige Expertise zusammenbringen. Netzwerke und Fachgemeinschaften als soziales Kapital und Kollektive mit geteilten
Interessen und Problemwahrnehmungen können dabei helfen, diese Expertise $\mathrm{zu}$ identifizieren und $\mathrm{zu}$ aktivieren, tragen aber auch dazu bei, dass sich fachbezogenes Wissen außerhalb von organisationalen Grenzen (wie bspw. Unternehmen) räumlich ausbreitet. Schließlich bieten Events (z.B. Festivals, Kongresse, Tagungen) Gelegenheiten für Akteure eines Felds wie Fachgemeinschaften, Märkte und Wettbewerber, sich für eine befristete Zeit an einem Ort zu treffen, sich auszutauschen und längerfristige Beziehungen aufzubauen (Maskell et al. 2004).

Diese strukturellen Veränderungen, verstärkt durch Prozesse der Digitalisierung und den technischen und organisatorischen Möglichkeiten des Internets (z.B. in Form von Plattformökonomien), haben deutliche Auswirkungen auf die räumliche Organisation von Arbeit, insbesondere in urbanen Räumen (Schmidt 2019; Brown 2017). Die Fragmentierung in immer kleinere unternehmerische Einheiten (bis hin zum Individuum als kleinste ökonomische Einheit) führt zu einer Ausdifferenzierung der physisch-materiellen Umgebungen, von denen aus Erwerbsarbeit organisiert wird und schließt die eigene oder eine fremde Wohnung ebenso ein wie Transiträume (z. B. Flughäfen, Bahnhöfe, Flugzeuge wie auch Züge), Cafés und geteilte Arbeitsumgebungen wie zum Beispiel Coworking Spaces.

\section{Open Creative Labs - Mehr als Treffpunkte für Kreative}

Es zeichnet sich ein Widerspruch ab zwischen einer zunehmenden räumlichen und organisatorischen Fragmentierung und Individualisierung der Erwerbsarbeit auf der einen Seite und der Volatilität der Arbeit auf der anderen Seite, die sich ausdrückt in der Notwendigkeit, kontinuierlich temporäre Arbeitsverbünde einzurichten, wofür soziales Kapital, Zugang zu potenziellen Kunden oder Auftraggebern wie auch Zugang zu und Verfügbarkeit von Arbeitsstrukturen notwendig ist. Zudem konzentrieren sich sämtliche unternehmerische Funktionen (Fachexpertise, administrative Aufgaben, Unternehmensstrategie, Marketing) auf immer weniger Individuen. Parallel zu dieser Entwicklung beobachten wir seit etwa 15 Jahren eine zunehmende Zahl an geteilten Arbeitsumgebungen (Schmidt 2019) wie Coworking Spaces oder Makerspaces, deren steigende Nachfrage vor dem Hintergrund der oben skizzierten Entwicklungen erklärt werden kann (Brown 2017; Merkel 2019; Blagoev et al. 2019).

Einige Beiträge verweisen darauf, dass diese geteilten Umgebungen als neuartige „Dritte Orte“ beschrieben werden können, in denen sich multiple Praktiken zwischen Arbeit und Freizeit etablieren und sich ausdrücken im Experimentieren, Arbeiten und spielerischem Umgang mit Ideen und Materialien (Brown 2017; Waters-Lynch und 


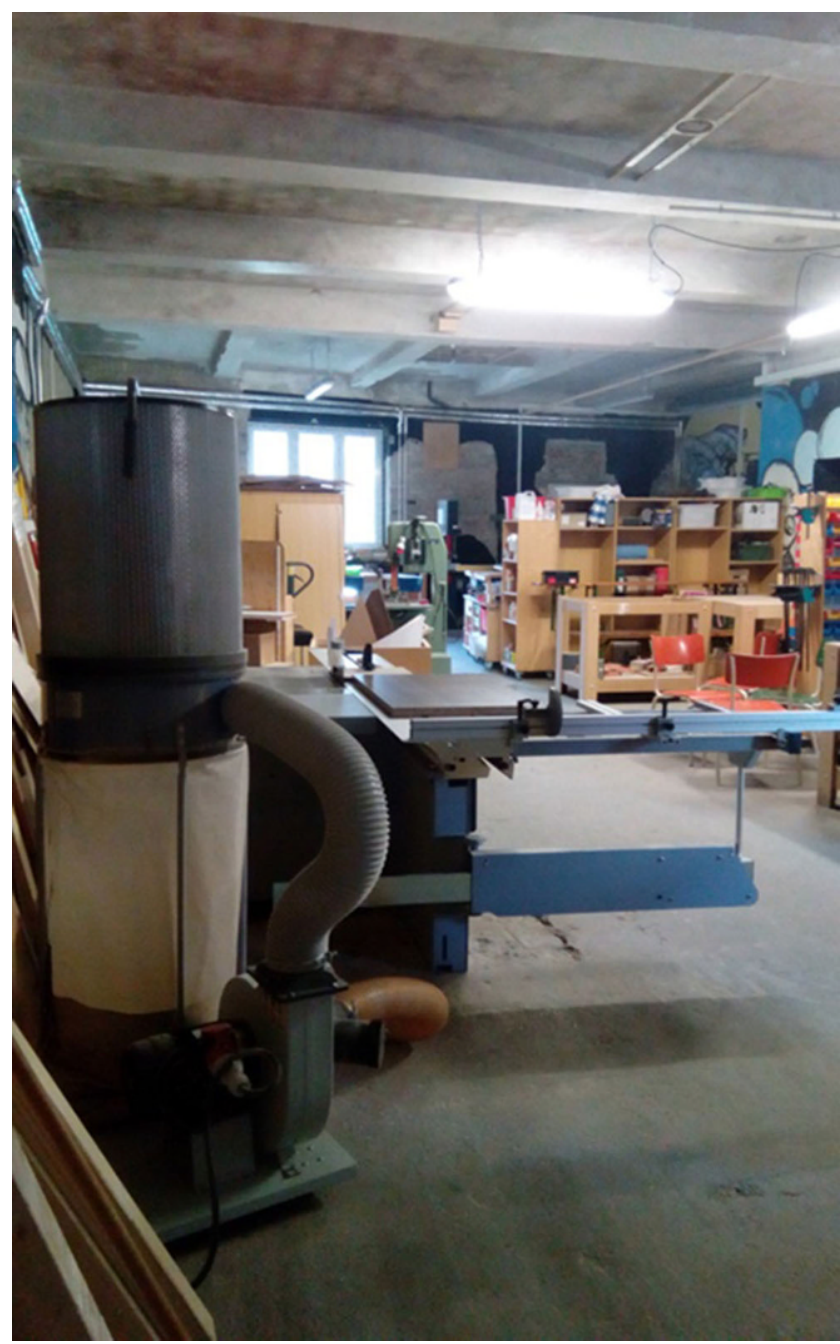

Abb. 2 Makerspace als geteilte Werkstatt. (@ Suntje Schmidt)

Potts 2017; vgl. auch Abb. 2). Ein genauerer Blick in die inzwischen breit ausgefächerte Bandbreite dieser Orte verdeutlicht jedoch, dass insbesondere geteilte Arbeitsumgebungen zwar viele Elemente von „Dritten Orten“ in sich vereinen, sich aber auch deutlich von ihnen unterscheiden und damit mehr als ,nur“ Treffpunkte für Kreative darstellen.

Bisher hat sich noch keine durchgängig geteilte Definition dieser neuen Umgebungen durchgesetzt, weswegen einige Autoren Sammelbegriffe für sie verwenden, beispielsweise kollaborative Arbeitsorte (,collaborative working spaces“; de Vaujany et al. 2016) oder Open Creative Labs (Schmidt et al. 2016). Innerhalb der Gruppe von Open Creative Labs wird unterschieden zwischen Experimentierlabs und arbeitsbezogenen Labs. Erstere können als ,Dritte Orte“ zwischen Arbeit und Freizeit interpretiert werden, die unabhängig von Bildung oder Qualifizierung leicht zugänglich sind und in denen Enthusiasten, Hobbyisten, Bastler und Tüftler aufeinandertreffen. Experimentierlabs können folglich Kristallisationskerne bilden für Gemeinschaften, die Interessen und Hobbies teilen.

Open Creative Labs als Arbeitsorte hingegen unterscheiden sich sehr deutlich von „Dritten Orten“, denn sie werden für Tätigkeiten genutzt, die im Zusammenhang mit der Erwerbsarbeit ihrer Nutzerinnen und Nutzer stehen. Gleichzeitig unterscheiden sie sich deutlich von traditionellen Arbeitsorten. So kann der Zugang zu ihnen flexibel gestaltet werden und ist eher zu vergleichen mit einer Eintrittskarte (für mehrere Stunden, Tage oder Wochen) in eine Arbeitsumgebung anstelle von fixen Kosten für beispielsweise mittelfristige Büromieten. Auch hierin ist ein deutliches Unterscheidungsmerkmal im Vergleich zu „Dritten Orten“ zu sehen, die in der Regel frei zugänglich sind, wie z. B. Cafés oder Bibliotheken. Open Creative Labs als Arbeitsumgebungen bieten eine Umgebung für die Umsetzung der erwerbsbezogenen Ideen und Projekte ihrer Nutzerinnen und Nutzer. Obwohl folglich keine eindeutige Nutzung a priori vorgeschrieben ist, ergibt sich ein labbezogener Schwerpunkt aus den Projekten der Nutzer und dem sozialen Kuratieren dieser Orte durch die Betreiber und vorhandenen Nutzergemeinschaften. Auch hierin ist eine $\mathrm{Zu}$ gangsbeschränkung zu sehen, denn damit sind implizite wie auch explizite Regeln für den Zugang zu Open Creative Labs verbunden. So lassen einerseits Ausstattungsmerkmale nur eine bestimmte Bandbreite an Nutzung zu. Gleichzeitig können vorhandene Nutzerinnen und Nutzer anziehend wirken oder eher den Eindruck erwecken, als neue Nutzerin/neuer Nutzer weniger passfähig in einem Lab zu sein. Explizite Regeln drücken sich zum Beispiel in offen formulierten Erwartungen aus. So wird beispielsweise in der Regel erwartet, dass Nutzerinnen und Nutzer offen dafür sind, angesprochen zu werden und ihr Wissen und ihre Fähigkeiten mit anderen Nutzerinnen und Nutzern zu teilen (Schmidt et al. 2016).

Hierin deutet sich an, dass Open Creative Labs in ihrem Wert aus der Perspektive ihrer Nutzerinnen und Nutzer weit über Treffpunkte hinaus reichen. In Open Creative Labs treffen zwar unterschiedliche Nutzerinnen und Nutzer aufeinander, aber eher im Sinne von zufälligen, wertvollen Begegnungen (Serendipität; Olma 2012, 2016), aus denen sich komplementäre Verbindungen ergeben können, beispielsweise in Form von gegenseitiger Unterstützung, Peer-Beratungen oder gar Marktbeziehungen. Daher werden diese Begegnungen auch inszeniert, zum Beispiel indem sichtbar gemacht wird, welche Veranstaltungen demnächst im Lab stattfinden oder über Visitenkarten die anwesenden Nutzerinnen und Nutzer (vgl. Abb. 3). Darüber hinaus können Open Creative Labs als Organisationsformen außerhalb von Organisationen interpretiert werden (Schmidt und Brinks 2017). Sie übernehmen Management und Koordinierungsfunktionen, über die z. B. Selbstständige oder junge Startups Zugang zu Märkten erlangen, oder aber die Vorzüge 
Abb. 3 Coworking Space als geteilte Büroumgebung. (C) Suntje Schmidt)

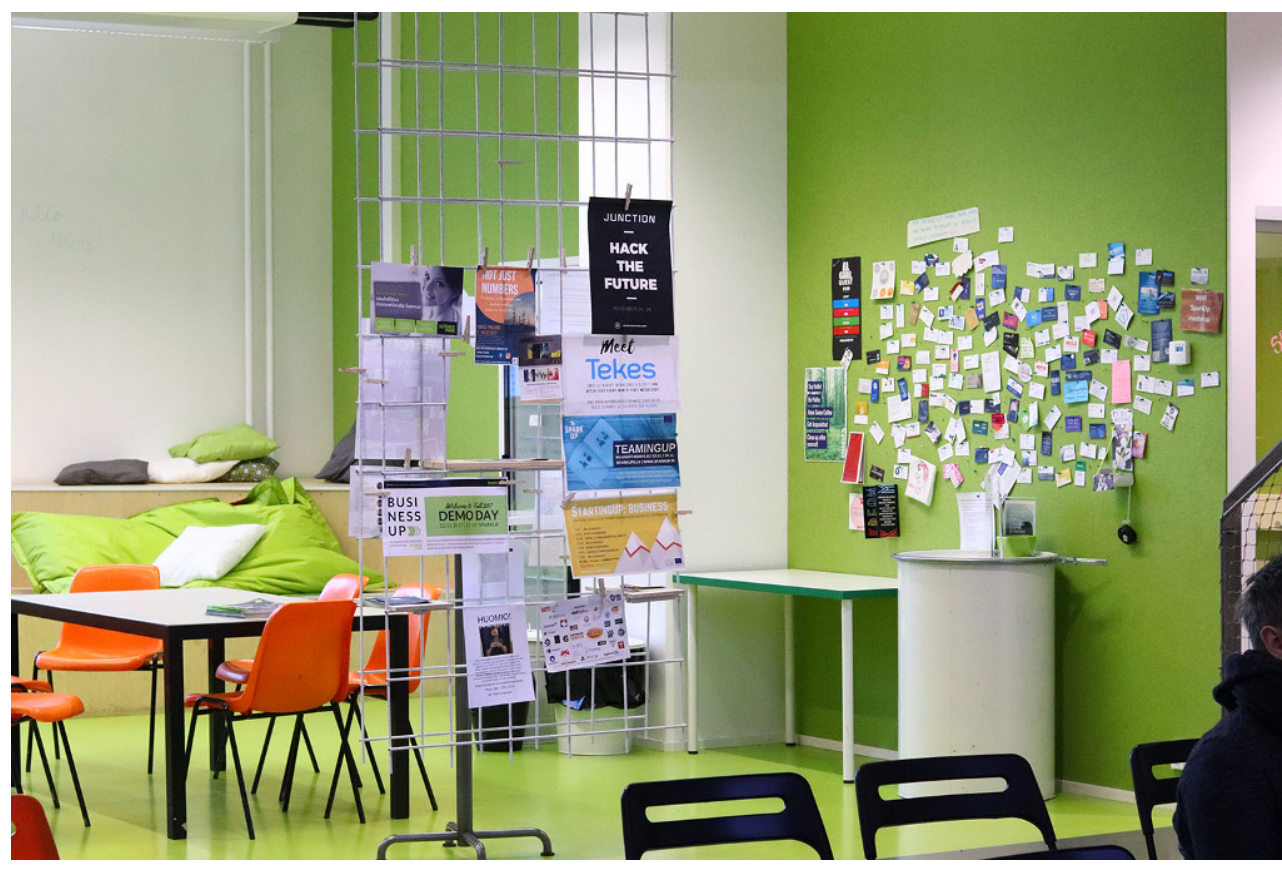

unabhängiger Kreativarbeit verbunden werden mit traditionellen Arbeitsstrukturen (Reuschl und Bouncken 2017). Hierbei spielen die Manager bzw. Betreiber von Labs eine Schlüsselrolle (Merkel 2015), denn sie verbinden ihre Labs - und damit ihre Nutzergemeinschaft - mit dem institutionellen und wirtschaftlichen Umfeld ihrer Labs (Ivaldi et al. 2018). Das bedeutet, dass sie gezielt Interaktionen zwischen individuellen Erwerbstätigen, Nutzergemeinschaften der Labs und Organisationen (wie z.B. Unternehmen) gestalten. Eine derartige gestalterische Funktion ist bei „Dritten Orten“ so nicht zu finden.

\section{Fazit}

Der wirtschaftliche Strukturwandel ist eng verbunden mit tiefgreifenden Wandlungsprozessen auf Arbeitsmärkten. Diese drücken sich in einem steigenden Anteil selbstständig Tätiger in wissensintensiven, kreativen Tätigkeitsbereichen aus. Organisatorisch verfasst sind diese Tätigkeiten oft in kleinteiligen Organisationseinheiten wie (Solo-)Selbstständigkeiten, Start-ups und freien Berufen. Diese organisatorische und räumliche Fragmentierung der Arbeit lässt den Bedarf nach neuen physischen und sozialen Räumlichkeiten steigen, für die Open Creative Labs die notwendigen Umgebungen anbieten. Aufgrund ihrer scheinbaren Offenheit und der gemeinschaftsstiftenden Funktionen werden Open Creative Labs - wie z. B. Coworking Spaces, Makerspaces, Innovationslabs - von einigen Autoren als „Dritte Orte" konzipiert, die Treffpunkte unabhängig vom sozialen Status darstellen und zugänglich für jeden sind. In seiner originären Definition sind „Dritte Orte“ Lokalitäten neben
Wohn- und Arbeitsorten, die sich einer derartig eindeutigen Funktionszuschreibung entziehen.

Im Gegensatz dazu etablieren sich Open Creative Labs jedoch als Arbeitsorte für eine steigende Zahl unabhängiger Kreativschaffender. Sie übernehmen Koordinierungs- und Managementfunktionen, bieten Arbeitsstrukturen und -umgebungen an und ermöglichen potenziell wertvolle Begegnungen. Damit reichen sie weit über Treffpunkte als „Dritte Orte" hinaus, mehr noch, sie etablieren sich als neuartige materielle und soziale Strukturen in modernen Arbeitswelten.

Danksagung Ich danke meinen Kolleginnen und Kollegen (Oliver Ibert, Verena Brinks, Andreas Kuebart, Steffi Brewig) für den inspirierenden Austausch, durch den viele wertvolle Ideen entstanden sind.

Förderung Die Forschungsarbeiten, die diesem Beitrag zugrunde liegen, sind mit Kolleginnen und Kollegen am Leibniz-Institut für Raumbezogene Sozialforschung entstanden (IRS), durch dessen Grundhaushalt das Projekt finanziert wurde.

Funding Open Access funding provided by Projekt DEAL.

Open Access Dieser Artikel wird unter der Creative Commons Namensnennung 4.0 International Lizenz veröffentlicht, welche die Nutzung, Vervielfältigung, Bearbeitung, Verbreitung und Wiedergabe in jeglichem Medium und Format erlaubt, sofern Sie den/die ursprünglichen Autor(en) und die Quelle ordnungsgemäß nennen, einen Link zur Creative Commons Lizenz beifügen und angeben, ob Änderungen vorgenommen wurden.

Die in diesem Artikel enthaltenen Bilder und sonstiges Drittmaterial unterliegen ebenfalls der genannten Creative Commons Lizenz, sofern sich aus der Abbildungslegende nichts anderes ergibt. Sofern das betreffende Material nicht unter der genannten Creative Commons Lizenz steht und die betreffende Handlung nicht nach gesetzlichen Vorschrif- 
ten erlaubt ist, ist für die oben aufgeführten Weiterverwendungen des Materials die Einwilligung des jeweiligen Rechteinhabers einzuholen.

Weitere Details zur Lizenz entnehmen Sie bitte der Lizenzinformation auf http://creativecommons.org/licenses/by/4.0/deed.de.

\section{Literatur}

Blagoev B, Costas J, Kärreman D (2019) 'We are all herd animals': Community and organizationality in coworking spaces. Organization. https://doi.org/10.1177/1350508418821008

Brown J (2017) Curating the "Third Place"? Coworking and the mediation of creativity. Geoforum 82:112-126. https://doi.org/10.1016/ j.geoforum.2017.04.006

Bundesagentur für Arbeit (2019) Strukturwandel nach Berufen. https:// statistik.arbeitsagentur.de/Navigation/Statistik/StatistischeAnalysen/Interaktive-Visualisierung/Strukturwandel-nachBerufen/Strukturwandel-nach-Berufen-Nav.html. Zugegriffen: 27. Dez. 2019

Castells M (2001) Der Aufstieg der Netzwerkgesellschaft: Das Informationszeitalter I - Die Netzwerkgesellschaft. Leske + Budrich, Opladen

de Vaujany F-X et al (2016) Collaborative communities in the city: From policies for to policies through third-places? https:// collaborativespacesstudy.wordpress.com/2016/04/10/rgcs- whitepaper-version-alpha/. Zugegriffen: 28. Dez. 2019

Florida R (2002) The rise of the creative class, and how it is transforming work, leisure, community and everyday life. Basic Books, New York

Florida R (2011) The rise of the creative class, revisited, 2. Aufl. Basic Books, New York

Florida R (2017) The new urban crisis: how our cities are increasing inequality, deepening segregation, and failing the middle class and what we can do about it. Basic Books, Hachette

Grabher G, Ibert O (2011) Project Ecologies. A contextual view on temporary organizations. In: Morris PWG, Pinot JK, Söderlund J (Hrsg) The Oxford Handbook of project management. Oxford University Press, Oxford, S 175-198

Houghton K, Foth M, Miller E (2013) The continuing relevance of the library as a third place for users and non-users of IT: The case of Canada Bay. Aust Libr J 62(1):27-39

IFB (2019) Entwicklung der Selbständigen in Freien Berufen in Deutschland, 1999-2019. http://ifb.uni-erlangen.de/wp-content/ uploads/Entwicklung-der-Selbstst\%C3\%A4ndigen-in-FreienBerufen-1999-2019.jpg. Zugegriffen: 18. Dez. 2019

Ivaldi S, Pais I, Scaratti G (2018) Coworking (s) in the plural: Coworking spaces and new ways of managing. In: Taylor S, Luckmann S (Hrsg) The new normal of working lives. Palgrave Macmillan; Springer, Cham, S 219-241

Maier MF, Ivanov B (2018) Forschungsbericht 514 - Selbständige Erwerbsarbeit in Deutschland. Bundesministerium für Arbeit und Soziales, ZEW, Mannheim
Manske A (2015) Kapitalistische Geister in der Kultur- und Kreativwirtschaft: Kreative zwischen wirtschaftlichem Zwang und künstlerischem Drang. transcript, Bielefeld

Maskell P, Bathelt H, Malmberg A (2004) Temporary clusters and knowledge creation: the effects of international trade fairs, conventions and other professional gatherings (SPACES online (2004-04))

Merkel J (2015) Coworking in the city. Ephemera Theory Polit Org 15(1):121-139

Merkel J (2019) 'Freelance isn't free.' Co-working as a critical urban practice to cope with informality in creative labour markets. Urban Stud 56(3):526-547. https://doi.org/10.1177/ 0042098018782374

Oldenburg R (1997) The great good place: Cafes, coffee shops, bookstores, bars, hair salons and other hangouts at the heart of the community. Da Capo Press, Cambridge

Oldenburg R, Brissett D (1982) The third place. Qual Sociol 5(4):265284

Olma S (2012) The serendipity machine: a disruptive business model for society 3.0. Lindonk \& De Bres, Amersfoort.

Olma S (2016) In defence of serendipity: for a radical politics of innovation. Repeater Books, London

Purnell D, Cunningham DB (2017) Traveling the third place: conferences as third places. Space Cult. https://doi.org/10.1177/ 1206331217741078

Reuschl AJ, Bouncken RB (2017) Coworking-Spaces als neue Organisationsform in der Sharing Economy. In: Bruhn M, Hadwich K (Hrsg) Forum Dienstleistungsmanagement. Dienstleistungen 4.0: Geschäftsmodelle - Wertschöpfung - Transformation, Bd. 2. Springer, Wiesbaden, S 185-208 https://doi.org/10.1007/978-3658-17552-8_8

Schmidt S (2019) In the making: open creative labs as an emerging topic in economic geography? Geogr Compass 13(9):1-16

Schmidt S, Brinks V (2017) Open creative labs: spatial settings at the intersection of communities and organizations. Creat Innov Manag 26(3):291-299. https://doi.org/10.1111/caim.12220

Schmidt S, Ibert O, Kuebart A, Kühn J (2016) Open Creative Labs: Typologisierung, Verbreitung und Entwicklungsbedingungen. Leibniz-Institut für Raumbezogene Sozialforschung. https://leibnizirs.de/fileadmin/user_upload/Transferpublikationen/2017-Open_ Creative_Labs_in_Deutschland-Typologisierung__Verbreitung_ und_Entwicklungsbedingungen.pdf. Zugegriffen: 28. Dez. 2019

Söndermann M, Strittmatter T (2007) Kultur- und Kreativwirtschaft im Land Brandenburg. Empirischer Grundlagenbericht zu den Strukturen und Entwicklungstrends des kulturwirtschaftlichen Sektors und des Non-profit-Kultursektors im Land Brandenburg. In: Tischvorlage zur Sitzung am 20.11.2007 im Auftrag des Ministeriums für Wirtschaft und des Ministeriums für Wissenschaft, Forschung und Kultur des Landes Brandenburg, Potsdam

Waters-Lynch J, Potts J (2017) The social economy of coworking spaces: a focal point model of coordination. Rev Soc Econ 75(4):417-433. https://doi.org/10.1080/00346764.2016.1269938 